\title{
Identité subjective du croyant et libre détermination des communautés religieuses en droit de l'Union européenne
}

\author{
Françoise CURTIT \\ DRES - Droits et religions, CNRS / Université de Strasbourg
}

Poursuivant la création d'un marché unique tout en œuvrant à la construction d'un espace politique, l'Union européenne a vu s'accroître au fil des décennies ses domaines d'intervention et ses compétences définies par les traités. Ceux-ci ne lui confèrent aucune attribution particulière en matière religieuse, mais plusieurs des actes juridiques de l'Union européenne vont cependant prendre en compte le fait religieux dans sa dimension individuelle ou institutionnelle.

Les normes supérieures de l'ordre juridique européen que sont les traités fondateurs et la Charte des droits fondamentaux affirment le respect des droits fondamentaux ${ }^{1}$, et en particulier de la liberté de religion ${ }^{2}$ et de la non-discrimination ${ }^{3}$. Par ailleurs, de nombreux textes pris dans les domaines de compétence de l'Union abordent la question religieuse, soit pour garantir là aussi la protection des convictions et le principe de non-discrimination, soit pour introduire des mesures dérogatoires ou spécifiques pour les organisations ou les activités confessionnelles. Ainsi, des dispositions éparses vont garantir le libre exercice du culte sous ses différentes formes (abattage rituel, diffusion des services religieux à la télévision...) ou préserver l'autonomie d'organisation des communautés religieuses et reconnaître le caractère d'intérêt général de certaines de leurs activités (en matière de droit du travail, de traitement de données personnelles, de fiscalité... $)^{4}$. Il s'agit dans la plupart des cas de mesures ponctuelles qui sont largement laissées à l'appréciation des États membres, les textes concernés envisageant explicitement la possibilité pour ceux-ci de prévoir ou non des exceptions ou de maintenir des dispositions plus favorables. L'article $17 \mathrm{du}$ Traité sur le fonctionnement de l'Union européenne (TFUE) affirme en effet le respect par l'Union européenne des régimes nationaux des cultes qui demeurent du ressort des États membres. Les dispositions du droit de l'Union européenne relatives aux questions religieuses s'attachent ainsi à préserver les pratiques et législations nationales existantes lorsqu'elles sont susceptibles d'être touchées par le droit européen dans l'un ou l'autre de ses domaines d'intervention.

Les dispositions européennes mentionnant le fait religieux concernent d'une part la prise en compte de certaines conduites ou pratiques des individus liées à - ou fondées sur - la religion ou les convictions et, d'autre part, l'organisation des institutions religieuses et leurs activités notamment sociales. Ces mesures ne visent pas directement l'identité religieuse de l'individu et si elles évoquent parfois la prise en compte de la religion d'une personne, elles ne tentent jamais de définir les critères de son appartenance à une confession ou à une communauté. La directive 2011/95 du 13 décembre 2011 concernant le statut de réfugié est d'ailleurs l'un des rares textes de droit de l'Union européenne qui se hasarde à définir la notion de religion, qui recouvre ici « en particulier, le fait d'avoir des convictions théistes, non théistes ou athées, la participation à des cérémonies de culte privées ou publiques, seul ou en communauté, ou le

\footnotetext{
${ }^{1}$ Art. 6 du Traité sur l'Union européenne.

${ }^{2}$ Art. 10 de la Charte des droits fondamentaux de l'Union européenne.

${ }^{3}$ Art. 10 et art. 19 du Traité sur le fonctionnement de l'Union européenne; Art. 21 de la Charte des droits fondamentaux de l'Union européenne.

${ }^{4}$ Voir F. CURTIT, « Le droit de l'Union européenne : prise en compte des activités et organisations religieuses et affirmation des droits fondamentaux », in F. MESSNER, P.-H. PRELOT et J.-M. WOEHRLING (dir.), Traité de droit français des religions, 2 éd., Paris, LexisNexis, 2013, p. 525-557.
} 
fait de ne pas y participer, les autres actes religieux ou expressions d'opinions religieuses, et les formes de comportement personnel ou communautaire fondées sur des croyances religieuses ou imposées par ces croyances $\gg^{5}$. Cette définition tend à inclure tous les aspects des convictions religieuses - ou athées ${ }^{6}$ - et de leurs manifestations, sans cependant mentionner la notion d'appartenance ou d'affiliation religieuse en tant que telle.

La Cour de justice de l'Union européenne n'a quant à elle fait référence qu'à de rares occasions à la liberté de religion ${ }^{7}$ et s'est la plupart du temps contentée de juger les affaires présentant un aspect religieux à la lumière des dispositions communautaires liées à la réalisation du marché unique. Elle s'est cependant prononcée sur ce sujet en septembre 2012 à l'occasion de demandes de décision préjudicielle présentées par l'Allemagne concernant l'ancienne directive relative aux conditions d'octroi du statut de réfugié. La Cour précise notamment que «la circonstance subjective que l'observation d'une certaine pratique religieuse en public [...] est particulièrement importante pour l'intéressé aux fins de la conservation de son identité religieuse est un élément pertinent dans l'appréciation du niveau de risque auquel le demandeur serait exposé dans son pays d'origine du fait de sa religion, même si l'observation d'une telle pratique religieuse ne constitue pas un élément central pour la communauté religieuse concernée $\gg^{8}$. La Cour met en avant ici la vision subjective de l'individu qui, plutôt que la doctrine officielle de la confession ou les règles d'une organisation religieuse, est le mieux à même de déterminer ce qui constitue le noyau dur de sa croyance. Plutôt qu'aux notions d'appartenance ou d'affiliation religieuse entendues comme un lien d'adhésion institutionnelle ou d'acceptation réciproque entre un individu et une communauté de conviction, le droit de l'Union européenne va davantage faire référence à l'identité subjective du croyant et à son autonomie personnelle, éléments centraux de la liberté de religion. La libre organisation des communautés confessionnelles et leur droit à définir par elles-mêmes les conditions que doivent remplir ceux qu'elles acceptent comme membres sont par ailleurs garantis, mais ils ne sauraient prévaloir sur la liberté individuelle du croyant de fixer la teneur et les contours de sa foi, ces deux composantes de la liberté de religion devant faire l'objet d'un juste équilibre.

La jurisprudence de la Cour de justice étant fort rare en la matière, c'est essentiellement dans les textes juridiques que l'on peut tenter de déterminer le contenu de la protection des convictions et trouver quelques marqueurs des liens qui relient l'individu à une communauté de croyance. Ils s'expriment par des mesures dérogatoires pour les organisations à finalité religieuse et protectrices pour les individus, d'une part sous la forme du droit à l'affirmation de convictions sans discrimination et sans justification (I) et, d'autre part, en matière de traitement des données à caractère personnel (II).

\section{L'identité religieuse et le principe de non-discrimination en droit de l'Union européenne}

\footnotetext{
${ }^{5}$ Art. $10 \S 1$ b) de la directive 2011/95/UE du Parlement européen et du Conseil du 13 décembre 2011 concernant les normes relatives aux conditions que doivent remplir les ressortissants des pays tiers ou les apatrides pour pouvoir bénéficier d'une protection internationale, à un statut uniforme pour les réfugiés ou les personnes pouvant bénéficier de la protection subsidiaire, et au contenu de cette protection : JOUE L 337, 20 déc. 2011 , p. 9.

${ }^{6}$ Comme beaucoup d'organisations de droit international, l'Union européenne promeut un traitement égal des différentes confessions religieuses. Elle prône par ailleurs la prise en compte des convictions philosophiques et athées et des groupements philosophiques ou non confessionnels. Voir notamment art. 17\$2 TFUE.

${ }^{7}$ Voir notamment CJCE, 27 oct. 1976, Vivien Prais c/ Conseil des Communautés européennes : Rec. CJCE 1976, I, p. 1589, à propos d'un concours de recrutement du Conseil organisé un jour de fête religieuse.

${ }^{8}$ CJUE, 5 sept. 2012, aff. jointes C-71/11 et C-99/11, Bundesrepublik Deutschland c/ Y. et Z, point 70.
} 


\section{I.1. Des dérogations pour les organisations dont l'éthique est fondée sur la religion ou les convictions}

En droit de l'Union, le respect de la liberté de religion s'affirme principalement à travers le principe de non-discrimination sur le fondement de la religion ou des convictions qui correspond à une approche concrète et pragmatique de l'Union européenne, laquelle protège les droits fondamentaux en relation avec les objectifs déterminés par les traités : l'égalité de traitement entre les individus doit notamment participer à l'élimination de coûts économiques et sociaux qui nuisent à la réalisation du marché intérieur et entravent la cohésion sociale. Le principe de non-discrimination sur le fondement de la religion a été introduit en droit primaire en 1997 seulement et figure aujourd'hui aux articles 10 et 19 TFUE ainsi qu'à l'article 21 de la Charte des droits fondamentaux. Il s'applique aux institutions et organes de l'Union dans l'exercice de leurs compétences et aux États lorsqu'ils mettent en œuvre le droit de l'Union.

Dans le domaine de l'emploi (accès à l'emploi, conditions de travail, formation professionnelle, affiliation aux organisations professionnelles), la directive 2000/78 du 27 novembre $2000^{9}$ établit sur cette base juridique un cadre général pour lutter contre la discrimination «fondée sur la religion ou les convictions, l'handicap, l'âge ou l'orientation sexuelle » (article 1). Elle prévoit cependant la possibilité de maintenir des différences de traitement, en particulier pour certaines activités professionnelles des communautés religieuses. Les États membres peuvent ainsi maintenir des législations qui permettent aux «églises et autres organisations publiques ou privées dont l'éthique est fondée sur la religion ou les convictions » de prendre en compte la religion ou les convictions d'une personne lorsqu'elles constituent «une exigence professionnelle essentielle, légitime et justifiée eu égard à l'éthique de l'organisation ». Les organisations confessionnelles et les établissements qui leur sont liés peuvent ainsi recruter des collaborateurs sur la base de critères religieux sans que cela constitue une discrimination, et ce en raison de leur finalité particulière qui suppose une adhésion réelle des personnels, une «attitude de bonne foi et de loyauté envers l'éthique de l'organisation» (article 4). Les responsables d'une école confessionnelle peuvent par exemple recruter à titre préférentiel des enseignants qu'ils estiment adhérer parfaitement au point de vue religieux de l'établissement. Dans ce cas, ce sont les organisations elles-mêmes qui déterminent les facteurs qui doivent être conformes à leur doctrine, voire les éléments constitutifs de l'appartenance religieuse des individus. Elles ne peuvent agir néanmoins que dans le cadre d'une exigence professionnelle "essentielle, légitime et justifiée », ce qui conduit à réserver les dérogations au principe de non-discrimination aux seuls emplois liés très étroitement à l'éthique de l'organisation, sans pouvoir les généraliser à tous les personnels. Les États membres ont transposé cette directive de façon plus ou moins étendue en fonction de leurs pratiques antérieures, la Commission européenne veillant cependant à ce que les législations nationales demeurent strictement dans le cadre des objectifs définis par le texte ${ }^{10}$.

Le principe de l'autonomie des communautés religieuses sur le plan doctrinal et organisationnel est ainsi à l'origine d'un droit dérogatoire qui leur permet de déterminer les éléments constitutifs de l'identité religieuse qu'elles exigent de certains de leurs personnels, son application étant néanmoins rigoureusement encadrée afin de préserver les droits fondamentaux des individus.

\footnotetext{
${ }^{9}$ Directive 2000/78/CE du 27 novembre 2000 portant création d'un cadre général en faveur de l'égalité de traitement en matière d'emploi et de travail : JOCE L 303, 2 déc. 2000, p. 16.

${ }^{10}$ Voir par exemple la lettre de mise en demeure adressée par la Commission européenne à la République fédérale d'Allemagne concernant la transposition de la directive 2000/78 en droit interne (loi allemande sur l'égalité de traitement) qui ne respectait pas le caractère essentiel, légitime et justifié de l'exigence professionnelle fondant la différence de traitement : procédure $n^{\circ}$ 2007/2362, 31 janv. 2008 .
} 


\section{I.2. Une présomption d'appartenance}

Le principe de non-discrimination vise à lutter contre des inégalités de traitement qui seraient fondées, parmi d'autres critères, sur la religion ou les convictions d'une personne et à limiter l'éventuel impact de celles-ci sur une situation donnée. Pas plus que les États membres l'Union européenne ne va chercher à se prononcer sur l'authenticité de l'appartenance ou des croyances religieuses d'un individu pour veiller à garantir ce principe. Dans le cadre des travaux préparatoires de la directive 2000/78, un rapport du Parlement européen écarte ainsi la possibilité de définir plus précisément les bases de la discrimination en arguant que retenir telle religion ou conviction plutôt que telle autre reviendrait à pratiquer une «politique de reconnaissance » contraire au principe même d'égalité. Il y est précisé par ailleurs que "l'interdiction des discriminations concerne l'interdiction d'une distinction établie par une personne vis-à-vis d'une autre sur la base d'une caractéristique ou d'une particularité attribuée par la première à cette dernière. La question de savoir si l'intéressé possède réellement cette caractéristique ou particularité est en principe dénuée de pertinence ${ }^{11}$. En la matière, ce n'est pas la réalité de l'identité religieuse - l'identité avérée - qui est prise en compte, mais bien la présomption d'identité ou d'appartenance : un traitement discriminatoire peut résulter de ce qu'un individu $\mathrm{X}$ a pensé, cru, supposé... à propos de l'appartenance, des pratiques ou des croyances d'un individu Y, sans que ces conjectures aient besoin de se révéler fondées. Cette notion de présomption d'appartenance figure de façon explicite dans une proposition de directive $^{12}$ rédigée en 2008 , et toujours en cours de discussion faute d'accord entre les États membres, qui prévoit de compléter les dispositions actuelles en étendant l'interdiction de la discrimination à des secteurs autres que celui du marché du travail (protection sociale, avantages sociaux, éducation, accès aux biens et services y compris au logement). L'amendement 41 déposé par le Parlement européen a ainsi introduit un paragraphe 4bis à l'article 2 consacré au «concept de discrimination» qui précise que «la discrimination fondée sur des suppositions concernant la religion ou les convictions, le handicap, l'âge ou l'orientation sexuelle d'une personne [...] est considérée comme une discrimination au sens du paragraphe $1 \gg$.

En matière de lutte contre la discrimination fondée sur la religion, le droit de l'Union européenne va ainsi s'attacher aux comportements et pratiques concrètes au regard du principe de l'égalité de traitement, en matière d'accès à l'emploi notamment, sans s'intéresser à l'effectivité de l'identité ou de l'appartenance confessionnelle des individus concernés.

Exceptions prévues pour les organisations confessionnelles et primauté accordée aux droits individuels sont également des éléments centraux en matière de traitement des données à caractère personnel, domaine où la notion d'identité voire d'appartenance religieuse est évoquée de façon la plus manifeste, sans que là encore l'Union ne cherche à en définir ou préciser le contenu.

\section{L'identité religieuse et le traitement des données à caractère personnel}

\footnotetext{
${ }^{11}$ Parlement européen, Commission de l'emploi et des affaires sociales, Rapport sur la proposition de directive du Conseil portant création d'un cadre général en faveur de l'égalité de traitement en matière d'emploi et de travail (procédure Hughes), rapporteur : Thomas Mann, 21 sept. 2000 : A5-0264/2000, p. 53.

${ }^{12}$ Proposition de directive du Conseil relative à la mise en œuvre du principe de l'égalité de traitement entre les personnes sans distinction de religion ou de convictions, de handicap, d'âge ou d'orientation sexuelle : COM/2008/0426 final.

${ }^{13}$ Résolution législative du Parlement européen du 2 avril 2009 sur la proposition de directive du Conseil relative à la mise en œuvre du principe de l'égalité de traitement entre les personnes sans distinction de religion ou de convictions, de handicap, d'âge ou d'orientation sexuelle : P6_TA(2009)0211.
} 


\section{II.1. Des dérogations pour les organismes à finalité philosophique ou religieuse}

Intervenant dans le cadre du rapprochement des législations nationales qui ont pour objet l'établissement et le fonctionnement du marché intérieur (article 114 TFUE), plusieurs dispositions du droit de l'Union européenne garantissent la protection des données à caractère personnel lors de la constitution de fichiers manuels ou informatisés. Elles s'appliquent en particulier aux données relatives aux convictions religieuses ou philosophiques, tout en prévoyant un certain nombre d'exceptions pour les traitements mis en œuvre par les organisations confessionnelles.

Faisant l'objet d'articles spécifiques en droit primaire (notamment article 16 TFUE et article 8 de la Charte des droits fondamentaux), la protection des données à caractère personnel est reconnue en tant que droit fondamental autonome, étroitement lié au droit au respect de la vie privée et familiale (article 7 de la Charte des droits fondamentaux), mais distinct de ce dernier. On peut noter que de telles dispositions explicites visant la protection des données sont par ailleurs fort rares dans les textes de droit international.

Sur cette base, c'est la directive 95/46 du 24 octobre $1995^{14}$ qui garantissait jusqu'il y a peu la protection des personnes physiques à l'égard des traitements de données à caractère personnel en encadrant strictement leurs modalités. Prenant en compte le contexte d'internationalisation croissante des échanges numériques via Internet, l'élaboration d'un nouveau «Paquet sur la protection des données » lancé en janvier 2012 vise à doter l'Union d'un cadre juridique plus solide en la matière et à assurer une protection uniforme dans les différents États membres. Les travaux ont abouti au printemps 2016 à l'adoption d'une directive consacrée aux traitements relatifs à la justice et à la sécurité ${ }^{15}$ et d'un règlement ${ }^{16}$ applicable dans tous les autres domaines d'activité et abrogeant la directive 95/46 dont il actualise et développe les principes.

Le règlement 2016/679 énonce dans son article $9 \$ 1$ un principe général d'interdiction de traitements portant sur des catégories particulières de données sensibles, dont « le traitement des données à caractère personnel qui révèle [...] les convictions religieuses ou philosophiques [...] d'une personne physique ». Le même article prévoit cependant des exceptions à cette prohibition, en particulier pour certains traitements de données effectués par des organismes à but non lucratif et à finalité philosophique ou religieuse. Les contraintes de ces collectivités ont été prises en compte et, au même titre que les partis politiques ou les syndicats, elles bénéficient en tant qu'institutions de dispositions dérogatoires qui visent à faciliter leur organisation interne et leur fonctionnement administratif : on songe, par exemple, aux cultes qui collectent un impôt payé par leurs membres ou à certaines Églises dont les organes dirigeants sont élus par les fidèles et qui ont donc besoin de recenser ceux-ci en tenant compte, entre autres éléments, d'une affiliation de type associatif ou d'une appartenance religieuse définie selon les conceptions propres à chaque confession. Le règlement 2016/679 permet à ces organismes d'effectuer des traitements de données interdits par ailleurs, en les encadrant néanmoins de façon rigoureuse, puisqu'ils ne peuvent être

\footnotetext{
${ }^{14}$ Directive 95/46/CE du 24 oct. 1995 relative à la protection des personnes physiques à l'égard du traitement des données à caractère personnel et à la libre circulation de ces données : JOCE L 281, 23 nov. 1995, p. 31.

${ }^{15}$ Directive 2016/680 du Parlement européen et du Conseil du 27 avril 2016 relative à la protection des personnes physiques à l'égard du traitement des données à caractère personnel par les autorités compétentes à des fins de prévention et de détection des infractions pénales, d'enquêtes et de poursuites en la matière ou d'exécution de sanctions pénales, et à la libre circulation de ces données, et abrogeant la décision-cadre 2008/977/JAI du Conseil : JOUE L 119, 4 mai 2016, p. 89.

${ }^{16}$ Règlement 2016/679 du Parlement européen et du Conseil du 27 avril 2016 relatif à la protection des personnes physiques à l'égard du traitement des données à caractère personnel et à la libre circulation de ces données, et abrogeant la directive 95/46/CE : JOUE L 119, 4 mai 2016, p. 1.
} 
réalisés que «dans le cadre de leurs activités légitimes et moyennant les garanties appropriées » et «à condition que ledit traitement se rapporte exclusivement aux membres ou aux anciens membres dudit organisme ou aux personnes entretenant avec celui-ci des contacts réguliers en liaison avec ses finalités et que les données à caractère personnel ne soient pas communiquées en dehors de cet organisme sans le consentement des personnes concernées » (article $9 \S 2 \mathrm{~d}$.).

Ces dispositions reprennent de façon assez similaire celles de la directive 95/46 en étendant le champ d'application des traitements dérogatoires aux « anciens membres » de ces organismes. La notion de «membre» exprimée ici semble évoquer directement l'appartenance à une institution de type associatif, voire à une communauté plus informelle, elle n'a cependant été définie ni dans les travaux préparatoires au texte ni par la jurisprudence. À l'instar de la plupart des droits nationaux, le droit de l'Union européenne ne se prononce pas sur cette qualification et considère comme membres les individus que les organisations religieuses reconnaissent elles-mêmes comme tels, qu'elles se fondent sur des critères formels (inscription, paiement de cotisations...) ou d'origine doctrinale (sacrement, rituel, filiation, engagement individuel...). En outre, la possibilité de dérogation au principe d'interdiction de traitement des données sensibles ne saurait justifier tous types de traitements de données liés de près ou de loin à des activités religieuses ${ }^{17}$, lesquels doivent par ailleurs respecter les autres conditions de licéité énoncées dans le texte.

Directement applicable dans les États membres, le règlement 2016/679 est entré en vigueur le 24 mai 2016 et sera applicable à partir du 25 mai 2018, date d'effet de l'abrogation de la directive 95/46. Les règles énoncées par celle-ci ont été par le passé rappelées par la Commission européenne à propos de leur application dans deux cas d'espèce relatifs à l'identité religieuse.

\section{II.2. La protection des droits individuels}

Le premier cas concerne la mention de la religion sur les cartes d'identité en Grèce. En réponse à une question parlementaire ${ }^{18}$ concernant cette inscription, à l'époque obligatoire, sur les cartes d'identité des citoyens grecs, la Commission a estimé que le traitement des données sensibles autorisé en vertu de l'article 8 de la directive 95/46 restait soumis à des conditions de licéité générales, et notamment que l'article $6 \mathrm{c}$ ) de la directive dispose que les données à caractère personnel doivent être «adéquates, pertinentes et non excessives au regard des finalités pour lesquelles elles sont collectées et pour lesquelles elles sont traitées ultérieurement». Par ailleurs, étant donné que «la finalité d'une carte d'identité est l'identification de la personne en précisant notamment sa nationalité et que la religion ne peut en aucun cas être considérée comme élément d'identification d'une personne, la mention de la religion sur une carte d'identité, même avec le consentement explicite de la personne concernée, pourrait être considérée comme non adéquate, non pertinente et excessive par rapport à la finalité de la carte d'identité ». Il résulte de ces considérations que « la mention de la religion sur la carte d'identité nationale avec le consentement indubitable de son titulaire pourrait être contraire à la directive 95/46 ». La Commission rappelle ici que si l'appartenance religieuse d'un individu doit figurer sur un document, cette inscription n'est possible que si elle est adaptée et proportionnée à l'objectif visé, lui-même légitime. Cet objectif devra en tout état de cause être mis en balance avec le droit de l'individu au respect de sa vie privée et

\footnotetext{
${ }^{17}$ Voir en particulier CJCE, 6 nov. 2003, aff. C-101/01, Lindqvist : Rec. CJCE, p. 12971 : à propos de pages Internet mises en ligne par une catéchiste dans une paroisse suédoise qui comportaient des informations nominatives sur des bénévoles de la paroisse sans déclaration auprès de l'autorité et sans accord des personnes concernées : la Cour conclut à la violation de la loi suédoise transposant la directive 95/46.

${ }^{18}$ Question orale à la Commission n ${ }^{\circ} 56$ de A. Alavanos, 17 mai 2000, H-0456/00.
} 
à la protection de ses informations personnelles et se conformer aux garanties générales prévues par la directive 95/46, y compris lorsqu'il s'agit de traitements de catégories particulières de données prévus par l'article 8. Malgré la campagne nationale de protestation organisée par l'Église orthodoxe, le gouvernement grec a finalement supprimé l'indication confessionnelle sur les cartes d'identité à partir de 2001 en considérant qu'elle était contraire à la loi 2472/1997 transposant la directive, suivant en cela les recommandations de l'Union européenne en la matière.

L'autre question sur laquelle la Commission a eu à se prononcer a trait à une instruction publiée le 23 septembre 2009 par l'Inspecteur général chargé de la protection des données personnelles en Pologne (GIODO), instruction relative aux traitements de données effectués dans le cadre des activités de l'Église catholique ${ }^{19}$. Ce texte précise notamment que les apostats ne peuvent pas exiger que leurs données personnelles soient effacées des archives paroissiales. Le parlementaire à l'origine de la question estime que cette instruction privilégie les dispositions du code de droit canonique plutôt que celles de la loi du 29 août 1997 sur la protection des données personnelles applicable en Pologne et que l'impossibilité de supprimer des données constitue une violation des droits de l'homme. Dans sa réponse, la Commission européenne juge d'abord que la tenue des registres par les paroisses catholiques s'inscrit dans le cadre du droit communautaire et constitue un traitement de données à caractère personnel réglementé par la directive 95/46. Elle rappelle par ailleurs que «les personnes concernées dans cette affaire ont tous les droits prévus dans la directive tels que le droit à l'information sur la nature du traitement, le droit d'accéder à leurs propres données et le droit de s'opposer au traitement de leurs données personnelles $»^{20}$. La Commission confirme ici l'application des dispositions de la directive à des archives paroissiales, soit dans ce cas précis à des registres de baptême et non pas uniquement à des fichiers de type administratif tels que, par exemple, la liste des membres d'une communauté locale. Le parlementaire évoque dans sa question un éventuel droit pour les individus concernés de voir «effacer» la mention de leur nom dans les registres paroissiaux, aspect sur lequel la Commission ne se prononce cependant pas directement, se contentant de rappeler le droit d'opposition qui figure à l'article 14 de la directive et qui prévoit que toute personne a le droit de «s'opposer à tout moment, pour des raisons prépondérantes et légitimes tenant à sa situation particulière » à ce que des données la concernant fassent l'objet d'un traitement. Elle ne souhaite manifestement pas se prononcer sur la question de la légalité au regard de la directive de demandes de radiation des registres baptismaux, problématique qui a été réactivée lors des débats liés à la réforme du droit de l'Union européenne en matière de traitement des données à caractère personnel.

\section{II.3. Le " droit à l'oubli »}

Outre l'introduction d'un droit explicite de retrait de son consentement par la personne concernée par le traitement de données (article 7\$3) et le maintien d'un droit d'opposition pour certains traitements (article 21), le règlement 2016/679 énonce dans son article 17 un «droit à l'effacement», aussi nommé «droit à l'oubli » qui prévoit l'obligation pour le responsable du traitement de supprimer «dans les meilleurs délais » les données à caractère personnel concernant une personne physique et de faire cesser leur diffusion, notamment lorsque la personne concernée s'oppose au traitement et qu'aucun «motif légitime impérieux » ne justifie de les conserver, ou lorsqu'elle retire son consentement et qu'il n'existe pas d'autre fondement juridique au traitement. La directive 95/46 prévoyait déjà dans son article $12 \mathrm{~b}$ ) la possibilité de demander la rectification ou l'effacement de données incomplètes ou inexactes et l'article $6 \S 1$ pouvait par ailleurs justifier la destruction de

\footnotetext{
${ }^{19}$ Question écrite à la Commission de J. SENYSZYN, 22 févr. 2010, E-0877/10.

${ }^{20}$ Traduit par nous à partir de la version anglaise.
} 
données lorsque leur traitement n'avait plus de finalité légitime ${ }^{21}$. Considéré par certains comme une «innovation juridique importante ${ }^{22}$, le droit à l'oubli numérique énoncé dans le règlement va plus loin en reconnaissant à la personne concernée le droit de faire disparaitre toute information personnelle qu'elle ne souhaite plus voir affichée, qu'il s'agisse des informations contenues dans le fichier de stockage d'origine, mais aussi de toutes les copies disponibles et de leurs référencements dans les moteurs de recherche. Des exceptions sont cependant prévues pour permettre la conservation des données nécessaires à l'exercice du droit à la liberté d'expression et d'information, pour des motifs d'intérêt public dans le domaine de la santé publique, à des fins historiques, statistiques ou archivistiques ou de recherche scientifique, pour respecter une obligation légale sous certaines restrictions ou constater, exercer ou défendre des droits en justice (article 17§3).

Introduite pour tenir compte de la réalité d'Internet qui permet de diffuser massivement des données personnelles sur le web marchand ou sur les réseaux sociaux et de les relier entre elles, cette conception du droit à l'oubli numérique s'est heurtée à de nombreuses critiques qui considèrent qu'il doit être mis en balance avec le droit à la liberté d'expression et d'information, voire même qu'il pourrait entrer en conflit avec la liberté de religion. Ainsi, l'Inspecteur général chargé de la protection des données personnelles en Pologne a estimé que «certaines communautés religieuses s'inquiètent que ce droit permette à des individus de détruire ou de réécrire leur histoire religieuse $»^{23}$. Â nouveau, certaines Églises redouteraient que la possibilité ouverte aux individus de demander l'effacement de données les concernant puisse s'appliquer aux registres paroissiaux et créer ainsi une prérogative qui se heurterait aux principes doctrinaux des confessions concernées.

La proposition de règlement a fait l'objet de fortes controverses dans les différents États membres sur divers points. Les débats ont notamment été vifs de la part des entreprises fournisseurs et intermédiaires d'Internet qui se voient dans l'obligation de retirer de leurs serveurs les contenus sur lesquels un droit à l'effacement aura été exercé et de prendre «des mesures raisonnables, y compris d'ordre technique », en vue d'informer les tiers traitant ou dupliquant ces données de la demande d'effacement de tout lien vers celles-ci ainsi que de toute copie ou reproduction (article 17§2). Cette disposition leur semblait en effet difficile à mettre en œuvre techniquement étant donné la complexité des réseaux d'information et la difficulté à s'assurer qu'aucune copie ne subsiste sur un quelconque ordinateur. Après plusieurs versions remaniées du texte, l'article 17 encadre les demandes d'effacement de données et de déréférencement en confirmant les obligations du responsable du traitement visà-vis des tiers, tout en prévoyant un certain nombre d'exceptions qu'il conviendra de délimiter précisément. Le droit à l'oubli est effectivement reconnu, mais sa mise en œuvre demeure complexe dans une société où l'information est toujours plus abondante, instantanée et interconnectée.

Ces dispositions relatives au droit à l'oubli méritent d'être signalées car cette notion semblait cristalliser un certain nombre de craintes des communautés confessionnelles, alors même que les droits d'opposition et d'effacement en vigueur jusqu'alors ne paraissent pas avoir été appliqués dans les États membres pour justifier, par exemple, un droit à l'effacement d'inscriptions dans des registres paroissiaux. Parmi les réponses aux «questions fréquentes » qui lui sont posées, le responsable de la protection des données de la République d'Irlande

\footnotetext{
${ }^{21}$ Les dispositions de la directive 95/46 ont ainsi été interprétées par la CJUE comme permettant à une personne de réclamer la suppression de liens litigieux de la liste des résultats affichés à la suite d'une recherche effectuée sur Google : CJUE, gde ch., 13 mai 2014, aff. C-131/12, Google Spain SL et Google Inc. c/ Agencia Española de Protección de Datos et Mario Costeja González, en particulier pt. $100 \S 3$.

${ }^{22}$ CJUE, gde ch., 13 mai 2014, précit. : conclusions de l'avocat général M. N. JÄÄSKINEN, 25 juin 2013, pt. 110.

${ }^{23}$ W. WIEWIÓROWSKI, in European Union data protection reform: new fundamental rights garantees, 3rd annual FRA symposium, Vienna, 10 May 2012, p. 7 (traduit par nous).
} 
rappelle ainsi que si la législation nationale prévoit que les personnes peuvent demander que des données inexactes les concernant soient corrigées, l'acte de baptême est un enregistrement précis d'une cérémonie qui s'est tenue à une certaine date et qu'il n'existe aucun droit en vertu de la loi de modifier ou de supprimer cette inscription ${ }^{24}$. En France, la cour d'appel de Caen a estimé dans un arrêt du 10 septembre 2013 que le baptême est un évènement public "qui constitu[e] un fait dont la réalité historique n'[est] pas contestée ${ }^{25}$ et dont la transcription sur le registre paroissial ne porte pas atteinte à la vie privée du requérant, lequel demandait la suppression intégrale de sa consignation plutôt que la simple mention de reniement obtenue dans un premier temps. La cour considère que les registres de baptême relèvent bien de la catégorie des traitements non automatisés de données à caractère personnel et sont soumis en tant que tels aux dispositions de la loi «informatique et libertés » du 6 janvier 1978 modifiée notamment en 2004 pour transposer la directive 95/46 - dont les exigences légales, en particulier le droit d'accès et de rectification, ont été en l'espèce remplies. La mention demandée au préalable par l'intéressé relative au reniement de son baptême a été opérée et sa liberté de ne pas appartenir à la religion catholique est ainsi respectée «sans qu'il y ait lieu à effacement ou correction supplémentaire ». Ici, la garantie des droits fondamentaux de la personne - en particulier le respect de sa vie privée et la protection de ses données personnelles - est mise en balance avec le droit à la libre administration du culte catholique pour qui l'établissement et la conservation de ce type d'acte constituent « une finalité légitime ». Le droit pour l'individu à l'affirmation de son identité religieuse - ou athée - ne saurait ainsi être absolu et doit tenir compte notamment des droits collectifs reconnus aux institutions confessionnelles.

La nouvelle réglementation européenne en matière de protection des données ne semble donc pas devoir modifier fondamentalement le droit et les pratiques existants en matière de traitement de données relatives aux institutions et activités religieuses et les craintes qui ont pu être exprimées en la matière ne paraissent donc guère fondées. Les dispositions de la directive 95/46 qui prévoyaient des exceptions pour les traitements effectués par les organismes à finalité philosophique ou religieuse ont été maintenues dans le nouveau règlement 2016/679 (art. $9 \$ 2$ d) et un nouvel article a été introduit les concernant. L'article 91 intitulé «Règles existantes des églises et associations religieuses en matière de protection des données » prévoit en effet que, lorsque dans un État membre, des organisations religieuses appliquent, à la date d'entrée en vigueur du règlement, des règles relatives à la protection des personnes physiques à l'égard du traitement des données à caractère personnel, «elles peuvent continuer d'appliquer lesdites règles à condition de les mettre en conformité avec le présent règlement ». Elles sont par ailleurs soumises au contrôle d'une autorité indépendante, laquelle peut être spécifique (distincte de l'autorité nationale de contrôle) pour autant qu'elle réponde aux conditions prévues pour les autorités de contrôle indépendantes (indépendance, compétence, missions...). Cet article 91 vise à mettre fin à l'existence de réglementations propres à des communautés confessionnelles qui ne respecteraient pas le droit européen en matière de protection des données, hypothèse qui semble au demeurant assez peu attestée. Il tient compte également des pratiques et législations nationales, prise en compte rappelée par ailleurs dans le considérant 165 du règlement qui énonce que ce dernier « respecte et ne porte pas préjudice au statut dont bénéficient, en vertu du droit constitutionnel en vigueur, les églises et les associations ou communautés religieuses dans les États membres, tel qu'il est reconnu par l'article 17 du traité sur le fonctionnement de l'Union européenne ».

\footnotetext{
${ }^{24}$ Frequently asked questions -FAQ, 2.15 Can I request to have my baptismal records deleted?: http://www.dataprotection.ie/docs/Right-of-access--right-of-rectification/1238.htm [consulté le 31 août 2016. 2016].

${ }^{25}$ CA Caen, 10 sept. 2013, n 11/03427, voir l'article de P.-H. PRELOT dans ce même volume. Solution confirmée par la Cour de cassation : Cass. $1^{\text {re }}$ civ., 19 nov. $2014, \mathrm{n}^{\circ} 13-25156$.
} 
Cet article 91 du règlement n'a guère fait l'objet de commentaires par les responsables religieux et on peut estimer qu'il n'apportera pas de modifications significatives aux règles existantes. La réforme du cadre européen de protection des données vise en premier lieu à corriger certains abus et dérives suscités par le développement des échanges numériques et semble donc devoir être sans incidence sur les activités et organisations confessionnelles, même si ces dernières continueront certainement de surveiller avec attention l'évolution de la législation en la matière.

Le droit de l'Union européenne ne vise pas à produire ses propres standards pour définir l'appartenance ou l'affiliation religieuse des individus et privilégie en la matière leur libre arbitre et l'autonomie d'organisation des confessions. Il ne tente donc aucunement de définir les critères de l'adhésion à une structure organisationnelle ou de déterminer les conditions subjectives ou doctrinales du lien créé entre un individu et une communauté de conviction, éléments laissés à l'appréciation du croyant ou des confessions elles-mêmes. En droit européen, l'appartenance religieuse est soit une identité religieuse conçue comme le ralliement volontaire d'une personne susceptible de variations, d'abandons et de revirements, soit une qualité de membre que les Églises et communautés religieuses attribuent à des individus selon des critères qui leur sont propres.

La Cour de justice de l'Union européenne s'est jusqu'à présent très peu prononcée sur cette question. Dans la perspective d'une protection élevée des droits fondamentaux dans l'ordre juridique européen, on peut concevoir qu'elle soit amenée à l'avenir à préciser l'équilibre à atteindre entre la dimension individuelle et subjective de la religion et sa dimension collective, tout en devant les concilier avec les autres droits et libertés fondamentales garantis au niveau européen. 